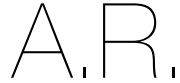

${ }^{1}$ Laboratório de Nutrição, Faculdade de Medicina, Universidade de Lisboa, Avenida Professor Egas Moniz,

1649-028 Lisboa, Portugal

2 Instituto de Saúde Ambiental, Faculdade de Medicina, Universidade de Lisboa,

Avenida Professor Egas

Moniz,

1649-028 Lisboa, Portugal

Endereço para correspondência:

Sofia Charneca

Laboratório de Nutrição,

Faculdade de Medicina

Universidade de Lisbo

Avenida Professor Egas Moniz,

1649-028

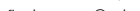

Histórico do artigo:

Recebido a 1 de setembro de 202 Aceite a 31 de dezembro de 202

\title{
SAÚDE MENTAL EM PERSPETIVA - O PAPEL DA NUTRIÇÃO E DA MICROBIOTA INTESTINAL
}

\author{
MENTAL HEALTH IN PERSPECTIVE - THE ROLE OF NUTRITION \\ AND GUT MICROBIOTA
}

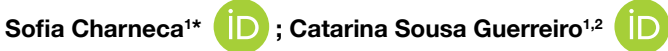

RESUMO

As perturbações mentais representam um dos mais importantes desafios da atualidade. No entanto, as opções terapêuticas disponíveis são nalguns casos pouco eficazes ou apresentam efeitos adversos importantes. As intervenções no âmbito do estilo de vida, onde se incluem intervenções nutricionais e alimentares, têm vindo a ser exploradas na área da doença psiquiátrica e já demonstraram exercer um efeito benéfico na sua otimização. Adicionalmente, existe uma conhecida associação entre a doença psiquiátrica e os distúrbios gastrointestinais. A evidência sugere que indivíduos com depressão apresentam uma composição da microbiota intestinal alterada, em comparação com indivíduos saudáveis. Neste sentido, também a microbiota intestinal poderá constituir um alvo terapêutico atrativo. Várias estratégias para a sua modificação já foram descritas, incluindo a manipulação da dieta, a utilização de suplementos probióticos e/ou prebióticos e o transplante de microbiota fecal. Pretende-se com este trabalho abordar importância da nutrição e alimentação no foro da psiquiatria, assim como a modulação da microbiota intestinal e utilização de psicobióticos, reconhecidos como uma potencial coadjuvante para o tratamento destas patologias. Conclui-se que, embora a informação atualmente disponível nesta matéria seja promissora, são necessários mais estudos para que seja possível formular recomendações específicas e cientificamente sustentadas.

\section{PALAVRAS-CHAVE}

Microbiota intestinal, Nutrição, Psicobióticos, Psiquiatria

\section{ABSTRACT}

Psychiatric disorders are some of the most serious challenges of our time. However, the currently available therapeutic options are often ineffective or carry severe side effects. Lifestyle interventions, which include dietary interventions, are being explored in the field of mental illness and have already been shown to exert beneficial effects. Additionally, there is a known association between psychiatric illness and gastrointestinal disorders, and patients with depression were reported to display an altered gut microbiota composition, when compared to healthy controls. In line with this, the gut microbiota may also be an attractive therapeutic target. Several approaches for its manipulation have already been described, such as dietary interventions, the use of probiotics and/or prebiotics and fecal microbiota transplantation. Thus, this review aims to summarize the importance of food and nutrition in the field of psychiatry, as well as the modulation of the gut microbiota and the use of psychobiotics, which have been recognized as a possible adjuvant therapy for these disorders. Finally, although current evidence in this matter is promising, further investigation is needed in order to translate this knowledge into clinical practice and make scientifically supported recommendations.

KEYWORDS

Gut microbiota, Nutrition, Psychobiotics, Psychiatry

\section{INTRODUÇÃO}

A saúde é definida pela Organização Mundial da Saúde, como um estado de completo bem-estar físico, mental e social e não apenas a ausência de doença ou enfermidade (1). Através desta definição é possível compreender que a saúde mental, durante anos negligenciada, é parte integrante da saúde e que se relaciona fortemente com a saúde física e com o comportamento, revelando-se essencial para o bem-estar dos indivíduos, e das sociedades em geral (2, 3). A nível nacional, as perturbações psiquiátricas têm uma prevalência de 22,9\%, uma das mais elevadas da Europa, representando assim um importante desafio da atualidade (4).
A ansiedade, a depressão e o stress estão intrínsecas nas sociedades modernas em que vivemos, e as intervenções no estilo de vida já demonstraram ser eficazes na sua atenuação (5). Embora as doenças psiquiátricas sejam alguns dos mais sérios desafios médicos e sociais da atualidade, a causa da maioria destas permanece desconhecida e as opções terapêuticas disponíveis são frequentemente pouco eficazes e/ou apresentam efeitos adversos graves (6). Os modelos de intervenção existentes no âmbito dos cuidados de saúde na doença psiquiátrica não conseguem responder na totalidade às complexas dimensões biológicas, sociais, culturais e 
espirituais da doença mental, tornando-se necessária a exploração de novos conceitos e opções terapêuticas (7). A nutrição e alimentação, enquadradas nos fatores associados ao estilo de vida, constituem alvos terapêuticos modificáveis, tendo vindo a ganhar um papel de destaque tanto na prevenção como no tratamento destas patologias (8-11).

A microbiota intestinal, cuja relevância em diversas patologias do trato digestivo está bem documentada (12), tem sido alvo de estudo nos mais diversos contextos. Neste sentido, tem surgido, nos últimos anos, um crescente corpo de evidência sugerindo que a microbiota intestinal tem também a capacidade de interagir com o sistema nervoso central e, por isso, poderá influenciar o comportamento e a função cerebral $(13,14)$. É atualmente conhecida uma associação entre a doença psiquiátrica e os distúrbios gastrointestinais (15), que será alvo de exploração com este trabalho.

\section{OBJETIVOS}

O presente trabalho tem como objetivos compilar a informação mais atual acerca do papel da nutrição e alimentação na prevenção e desenvolvimento das doenças psiquiátricas, assim como o impacto da modulação da microbiota intestinal como potencial alvo terapêutico. Pretende-se com esta revisão narrativa abordar o desenvolvimento atual desta temática, assim como sensibilizar os profissionais de saúde, no geral, e o nutricionista, em particular, para a mesma.

\section{METODOLOGIA}

Foi realizada uma pesquisa bibliográfica com recurso à base de dados PubMed, de artigos publicados entre 2011-2021. Foram incluídos artigos originais realizados em humanos (incluindo ensaios clínicos aleatorizados) e em modelos animais, assim como artigos de revisão, revisão sistemática e meta-análise, utilizando como critérios de pesquisa as palavras-chave "psychiatry and nutrition", "psychiatry and microbiota", "probiotics and prebiotics" e "psychobiotics". De acordo com a sua atualidade e pertinência para o tema, foi selecionada uma amostra de artigos cujo texto integral se encontrava disponível na língua portuguesa ou inglesa, de acesso gratuito.

\section{Nutrição, Doença Psiquiátrica e Microbiota Intestinal} O Conceito de Nutrição Psiquiátrica

O ramo da nutrição psiquiátrica oferece uma possível abordagem para uma gestão mais eficaz e minimização do impacto global associado à doença mental (16). A evidência sugere que a importância dos hábitos alimentares no contexto das doenças crónicas não transmissíveis também se estende à doença mental, tendo este facto sido reiterado no estudo de Jacka et al. no qual se verificou a existência de uma associação entre a prática de um padrão alimentar do tipo ocidental e uma maior probabilidade de ocorrência de sintomas e distúrbios psicológicos (17). Particularmente na depressão, um estudo realizado com uma amostra representativa da população portuguesa reportou que a prática de um padrão alimentar definido como não saudável, caracterizado por uma menor frequência de consumo de sopa, hortofrutícolas, peixe, leite/laticínios, menor ingestão de água e maior frequência de consumo de carne estava significativamente associada à presença de sintomas de depressão (18).

No âmbito da intervenção nutricional, deve ser privilegiada a obtenção de nutrientes através da prática de uma alimentação adequada, podendo também ser considerada a prescrição de nutrientes (nutracêuticos) suplementares $(19,20)$. São vários os nutrientes que apresentam uma ligação clara com a saúde e função cerebral e que podem ser considerados para este tipo de intervenção, nomeadamente os ácidos gordos polinsaturados $n-3$, as vitaminas do complexo B (como o folato e a vitamina B12), o ferro, o magnésio, a vitamina $D$, os aminoácidos, entre outros (19).

Ademais, considerando a alimentação como um todo ao invés dos seus nutrientes de forma isolada, a Dieta Mediterrânica (DM) destaca-se como uma estratégia que poderá ser útil em indivíduos com sintomas depressivos (21). Nesta temática, o estudo PREDIMED concluiu que indivíduos com diabetes mellitus tipo 2 que praticavam uma DM com frutos oleaginosos, numa porção de 30 gramas por dia (22), obtiveram uma redução de $40 \%$ no risco de depressão, comparativamente ao grupo placebo (23). Mais recentemente, o estudo HELFIMED comprovou o efeito benéfico de uma dieta do tipo mediterrânico suplementada com óleo de peixe (900 mg de DHA e 200 mg de EPA por dia (24)) em indivíduos com depressão (25). Por fim, o estudo SMILES (26) enfatiza a relevância da otimização da dieta como uma estratégia de tratamento eficaz e acessível para episódios depressivos major, referindo também que os benefícios desta intervenção se podem estender ao controlo de outras comorbilidades, o que é relevante pelo facto de as doenças mentais estarem associadas a um risco aumentado de obesidade, diabetes mellitus e doenças cardiovasculares (27). Ainda em relação às perturbações depressivas, foi verificada uma associação entre a prática de uma dieta pró-inflamatória (ocidental) e um risco aumentado de diagnóstico ou sintomas de depressão, comparativamente aos indivíduos que praticavam uma dieta anti-inflamatória (p.ex. DM) (28). Em concordância, uma revisão sistemática e meta-análise publicada em 2014 na revista American Journal Clinical Nutrition sugere que um padrão alimentar saudável, rico em hortofrutícolas, cereais integrais, peixe, carne de aves e produtos lácteos com baixo teor de gordura, poderá estar associado a um menor risco de depressão e apresenta um potencial para se afirmar como uma estratégia de prevenção primária para as perturbações depressivas (29). Esta informação vai ao encontro a algumas recomendações descritas na literatura para a prevenção da depressão, que incluem também a adoção de padrões alimentares tradicionais (onde se inclui a DM), o aumento do consumo de leguminosas, frutos oleaginosos e sementes, a inclusão de alimentos ricos em ácidos gordos polinsaturados n-3 e a limitação do consumo de alimentos processados, fast food e alimentos açucarados no geral (30). A insegurança alimentar é também um tópico importante a abordar neste contexto, uma vez que poderá afetar a saúde mental dos indivíduos, exercendo um efeito significativo na probabilidade de um indivíduo experienciar stress ou depressão (31), o que realça a importância da intervenção do Nutricionista nas suas diferentes valências. Finalmente, ainda relacionado à intervenção nutricional nesta população, devem ainda ser tidos em consideração os efeitos secundários da medicação com psicotrópicos, nomeadamente o aumento do apetite, aumento da ingestão alimentar ou compulsão alimentar (32-34).

\section{A Microbiota Intestinal, a Sua Relação com a Doença Mental e o Eixo Intestino-cérebro}

O ser humano alberga e coexiste com a microbiota intestinal, um ecossistema microbiano complexo que se estabelece essencialmente na porção mais distal do intestino (35). O intestino contém também mais de 100 milhões de neurónios e é, em última instância, um ponto de encontro de nervos, células imunológicas e microrganismos (36). Neste sentido, existe um complexo sistema de comunicação entre o trato gastrointestinal, os microrganismos que o habitam, e o sistema nervoso periférico e central (37). Esta rede de comunicação bidirecional de moléculas e sinais neurais, que engloba o trato gastrointestinal, o sistema nervoso e o cérebro, é denominada de eixo intestino-cérebro $(38,39)$. Existem vários mecanismos pelos quais a microbiota intestinal poderá modular o comportamento, assim como o desenvolvimento e 
a função cerebral, nomeadamente através do sistema imunológico, endócrino e neurológico $(37,40)$. Sendo a comunicação entre o intestino e o cérebro bidirecional, também o cérebro é capaz de modular a microbiota intestinal, através de alterações na motilidade, secreção gastrointestinal ou permeabilidade intestinal (41).

Com relevância para esta temática, já foi documentado que a microbiota intestinal de indivíduos com depressão apresenta uma menor riqueza e diversidade, em comparação com a microbiota intestinal de indivíduos saudáveis $(42,43)$. No entanto, o estudo da microbiota intestinal através de trabalhos observacionais não permite aferir uma relação de causalidade. Neste âmbito, o estudo desenvolvido por Kelly et al. (42), verificou que um transplante de microbiota fecal de indivíduos com depressão em ratos induziu nestes características comportamentais e fisiológicas da depressão e comportamentos semelhantes à ansiedade, facto que sugere que a microbiota intestinal poderá desempenhar um papel causal no desenvolvimento de certas características da depressão, podendo assim representar um alvo terapêutico na prevenção e tratamento desta doença (42). Adicionalmente, não só a microbiota intestinal está associada à doença psiquiátrica em si, como também pode influenciar os efeitos terapêuticos e/ou adversos associados à terapêutica farmacológica utilizada no seu tratamento $(44,45)$. Desta forma, é possível compreender que a modulação da microbiota poderá ser um alvo terapêutico ao nível da saúde mental.

\section{A Modulação da Microbiota Intestinal como Coadjuvante do Tratamento das Doenças Psiquiátricas \\ Modulação da Microbiota Intestinal: Como e Porquê?}

A microbiota intestinal possui plasticidade na sua composição e função $(46,47)$. Neste sentido, várias estratégias para a sua modificação já foram descritas na literatura, como a manipulação da dieta, a utilização de probióticos e/ou prebióticos, bem como o transplante de microbiota fecal $(37,46)$. Existe uma íntima relação entre a alimentação, a microbiota e a saúde, sugerindo que, possivelmente, alterações da microbiota induzidas pelo tipo de dieta poderão influenciar o desenvolvimento e progressão da doença (48). O tipo de dieta tem a capacidade de modular a composição da microbiota intestinal, uma vez que determina a disponibilidade de substratos para o crescimento microbiano e influencia o trânsito e ambiente intestinal (49). Esta informação é relevante pois, considerando a informação já descrita ao longo da presente revisão, é possível especular que a modulação da microbiota intestinal poderá ser (pelo menos em parte) o mecanismo pelo qual uma intervenção nutricional possa exercer o seu papel na saúde mental dos indivíduos.

Os probióticos, ou seja, "microrganismos vivos que, quando administrados em quantidades adequadas, conferem um benefício à saúde do hospedeiro" (50), também poderão afetar a microbiota intestinal, através da competição por nutrientes ou por locais de adesão à parede intestinal, da produção de substratos de crescimento para outras bactérias, entre outros (51). Os prebióticos, "substratos que são utilizados seletivamente por microrganismos do hospedeiro conferindo um benefício à saúde" (52), têm também a capacidade de modular a microbiota intestinal, ao estimularem seletivamente o seu crescimento e/ou atividade (53). Por último, o transplante de microbiota fecal, que consiste na transferência de bactérias fecais de um dador saudável para um indivíduo doente, também representa um método com a capacidade de modular a microbiota intestinal e tratar a disbiose, podendo ainda ser um método promissor em perturbações psiquiátricas resistentes ao tratamento (54). O seu uso potencial em transtornos psiquiátricos resistentes ao tratamento surge devido ao racional teórico relacionado com a restauração da microbiota intestinal alterada nestes doentes, não existindo ainda estudos realizados nesta população, de acordo com o nosso conhecimento. Relativamente ao estudo do efeito de probióticos e prebióticos neste contexto já foram realizados vários trabalhos (55). Alguns exemplos serão detalhados na próxima secção do presente artigo.

\section{A Era dos Psicobióticos}

O termo psicobiótico foi definido inicialmente, por um grupo de investigadores da University College Cork na Irlanda, como sendo um organismo vivo que, quando ingerido em quantidades adequadas, produz um benefício para a saúde em indivíduos que sofrem de doenças psiquiátricas (56). Posteriormente, a definição de psicobiótico foi reformulada e passou a incluir duas novas dimensões: em primeiro lugar, devido os benefícios dos psicobióticos em indivíduos saudáveis já documentados, a sua utilização não necessita de ser restrita a grupos clínicos; em segundo lugar, foram incluídos os prebióticos, por promoverem o crescimento de bactérias intestinais benéficas (38). Embora a definição de psicobiótico seja recente, o conceito de utilizar microrganismos como uma terapêutica adjuvante em doenças do espetro da psiquiatria tem sido discutido pela comunidade científica há vários anos. Na realidade, o primeiro estudo sobre esta matéria foi publicado em 1910 (57).

Diversos estudos de intervenção com psicobióticos já foram realizados desde então, inclusive em indivíduos saudáveis (58). Considerando populações clínicas, em indivíduos com perturbação depressiva major foi reportada uma diminuição significativa no score de depressão após 8 semanas de intervenção com uma cápsula de probióticos contendo Lactobacillus (L.) acidophilus, L. casei e Bifidobacterium (B.) bifidum (59). Em indivíduos com a mesma patologia, a toma de um probiótico contendo L. helveticus R0052 e B. longum R0175, também durante 8 semanas, resultou igualmente numa diminuição significativa do score de depressão, obtido através do Inventário de Depressão de Beck (60). Nesta temática, os resultados de uma revisão sistemática e meta-análise sobre o efeito dos probióticos no tratamento da depressão indicam que estes são eficientes na redução os sintomas desta doença apenas quando administrados em conjunto com a medicação antidepressiva (61). Em indivíduos com esquizofrenia, a suplementação vitamina D e de probióticos (L. acidophilus, B. bifidum, L. reuterie L. fermentum), durante 12 semanas, teve um efeito benéfico no perfil metabólico dos indivíduos e no score de uma escala utlizada para medir a severidade dos sintomas desta patologia, comparativamente ao placebo (62). Já em indivíduos com doença bipolar tipo 1 ou com perturbação esquizoafetiva do tipo bipolar que foram hospitalizados devido a um episódio maníaco, a toma de um probiótico durante 24 semanas, após receberem alta hospitalar, contendo L. rhamnosus $G G$ e $B$. animalis subsp. lactis Bb12 resultou numa menor taxa de re-hospitalização, comparativamente ao verificado nos indivíduos que receberam um placebo (63).

\section{ANÁLISE CRÍTICA}

As implicações das doenças do foro psiquiátrico vão muito além do que ocorre no cérebro. Naturalmente, para fazer face a uma doença que envolve várias dimensões da saúde, são necessárias respostas e intervenções de diferentes áreas. Os distúrbios gastrointestinais, a elevada prevalência de comorbilidades, os efeitos adversos da terapêutica farmacológica e a íntima relação entre o intestino e o cérebro são apenas alguns dos fatores que evidenciam e justificam a necessidade de intervenção de um nutricionista neste campo. Uma alimentação saudável e nutritiva em indivíduos com doença psiquiátrica pode comportar benefícios ao nível da saúde mental, além dos restantes benefícios já extensamente conhecidos. Na prática, de acordo 
com o abordado neste trabalho, recomenda-se uma dieta do tipo mediterrânica, representando esta um padrão alimentar não restritivo e culturalmente adequado à população portuguesa. Relativamente à utilização de psicobióticos, é impossível deixar de salientar que são muitas ainda as questões por responder, nomeadamente a definição exata de quais as estirpes bacterianas ou prebióticos com benefício na saúde mental, assim como qual a respetiva dosagem e duração da intervenção. É necessário também compreender de forma clara os mecanismos responsáveis pelos resultados observados e se estes estão dependentes da toma crónica de psicobióticos. Em suma, a informação disponível no momento é aliciante mas, embora existam diversos estudos que demonstram o seu efeito benéfico, estes são muito heterogéneos para que possam ser estabelecidas conclusões definitivas. Para que seja possível utilizar este conhecimento na prática clínica é crucial que sejam realizados mais estudos.

\section{CONCLUSÕES}

Não há dúvidas que ainda há muito por conhecer no âmbito da saúde mental e que é necessário continuar a trabalhar para o seu reconhecimento e tratamento adequado, que passa também por encontrar terapêuticas menos hostis que possam contribuir para o bem-estar e qualidade de vida dos indivíduos. A evidência sugere que a nutrição e alimentação, assim como a associação de psicobióticos, são intervenções com um caráter promissor na prevenção e atenuação do impacto das morbilidades do foro mental nas sociedades modernas.

\section{REFERÊNCIAS BIBLIOGRÁFICAS}

1. International Health Conference. Constitution of the World Health Organization. 1946. Bull World Health Organ. 2002;80(12):983-4.

2. World Health Organization. Promoting mental health: concepts, emerging evidence, practice: summary report/a report from the World Health Organization, Department of Mental Health and Substance Abuse in collaboration with the Victorian Health Promotion Foundation and the University. 2004.

3. World Health Organization. The World health report: 2001: Mental health: new understanding, new hope. 2001.

4. Conselho Nacional de Saúde. Sem mais tempo a perder - Saúde mental em Portugal: um desafio para a próxima década. Lisboa: CNS. 2019.

5. Rippe JM. Lifestyle Medicine: The Health Promoting Power of Daily Habits and Practices. Am J Lifestyle Med. 2018;12(6):499-512.

6. Sampson TR, Mazmanian SK. Control of brain development, function, and behavior by the microbiome. Cell Host Microbe. 2015;17(5):565-76.

7. Lake J, Turner MS. Urgent Need for Improved Mental Health Care and a More Collaborative Model of Care. Perm J. 2017;21:17-24.

8. Mörkl S, Wagner-Skacel J, Lahousen T, Lackner S, Holasek SJ, Bengesser SA, et al. The role of nutrition and the gut-brain axis in psychiatry: A review of the literature. Neuropsychobiology. 2018;17:1-9.

9. Sarris J, Logan AC, Akbaraly TN, Amminger GP, Balanzá-Martínez V, Freeman $M P$, et al. Nutritional medicine as mainstream in psychiatry. The Lancet Psychiatry. 2015;2(3):271-4.

10. Adan RAH, van der Beek EM, Buitelaar JK, Cryan JF, Hebebrand J, Higgs S, et al. Nutritional psychiatry: Towards improving mental health by what you eat. Eur Neuropsychopharmacol. 2019;29(12):1321-32.

11. Sarris J. Nutritional Psychiatry: From Concept to the Clinic. Drugs. 2019;79(9):929-34. 12. Nagao-Kitamoto H, Kitamoto S, Kuffa P, Kamada N. Pathogenic role of the gut microbiota in gastrointestinal diseases. Intest Res. 2016;14(2):127-38.

13. Cryan JF, Dinan TG. Mind-altering microorganisms: The impact of the gut microbiota on brain and behaviour. Nat Rev Neurosci. 2012;13:701-12.

14. Sandhu K, Sherwin E, Schellekens H, Stanton C, Dinan T, Cryan J. Feeding the microbiota-gut-brain axis: diet, microbiome, and neuropsychiatry. Transl Res. 2017;179:223-44.
15. North CS, Hong BA, Alpers DH. Relationship of functional gastrointestinal disorders and psychiatric disorders: Implications for treatment. World J Gastroenterol. 2007;13(14):2020-7.

16. Jacka FN. Nutritional Psychiatry: Where to Next? EBioMedicine. 2017;17:24-9. 17. Jacka FN, Pasco JA, Mykletun A, Williams LJ, Hodge AM, O'Reilly SL, et al. Association of western and traditional diets with depression and anxiety in women. Am J Psychiatry. 2010;167(3):305-11.

18. Gregório MJ, Rodrigues AM, Eusébio M, Sousa RD, Dias $S$, André B, et al. Dietary Patterns Characterized by High Meat Consumption Are Associated with Other Unhealthy Life Styles and Depression Symptoms. Front Nutr. 2017;4(25).

19. Sarris J, Logan AC, Akbaraly TN, Paul Amminger G, Balanzá-Martínez V, Freeman MP, et al. International Society for Nutritional Psychiatry Research consensus position statement: Nutritional medicine in modern psychiatry. World Psychiatry. 2015;14(3):370-1. 20. Firth J, Teasdale SB, Allott K, Siskind D, Marx W, Cotter J, et al. The efficacy and safety of nutrient supplements in the treatment of mental disorders: a meta-review of meta-analyses of randomized controlled trials. World Psychiatry. 2019;18(3):308-24. 21. Ventriglio A, Sancassiani F, Contu MP, Latorre M, Di Slavatore M, Fornaro M, et al. Mediterranean Diet and its Benefits on Health and Mental Health: A Literature Review. Clin Pract Epidemiol Ment Heal. 2020;16(Suppl-1):156-64.

22. Martínez-González MÁ, Corella D, Salas-salvadó J, Ros E, Covas MI, Fiol M, et al. Cohort profile: Design and methods of the PREDIMED study. Int J Epidemiol. 2012;41(2):377-85.

23. Sánchez-Villegas A, Martínez-González MA, Estruch R, Salas-Salvadó J, Corella D, Covas Ml, et al. Mediterranean dietary pattern and depression: The PREDIMED randomized trial. BMC Med. 2013;11:208.

24. Zarnowiecki D, Cho J, Wilson A, Bogomolova S, Villani A, Itsiopoulos C, et al. A 6-month randomised controlled trial investigating effects of Mediterranean-style diet and fish oil supplementation on dietary behaviour change, mental and cardiometabolic health and healthrelated quality of life in adults with depression (HELFIMED): st. BMC Nutr. 2016;2(1):1-10.

25. Parletta N, Zarnowiecki D, Cho J, Wilson A, Bogomolova S, Villani A, et al. A Mediterranean-style dietary intervention supplemented with fish oil improves diet quality and mental health in people with depression: A randomized controlled trial (HELFIMED). Nutr Neurosci. 2019;22(7):474-87.

26. Jacka FN, O'Neil A, Opie R, Itsiopoulos C, Cotton S, Mohebbi M, et al. A randomised controlled trial of dietary improvement for adults with major depression (the "SMILES" trial). BMC Med. 2017;15(1):23.

27. Firth J, Siddiqi N, Koyanagi A, Siskind D, Rosenbaum S, Galletly C, et al. The Lancet Psychiatry Commission: a blueprint for protecting physical health in people with mental illness. The Lancet Psychiatry. 2019;6(8):675-712.

28. Tolkien K, Bradburn S, Murgatroyd C. An anti-inflammatory diet as a potential intervention for depressive disorders: A systematic review and meta-analysis. Clin Nutr. 2019;38(5):2045-52.

29. Lai JS, Hiles S, Bisquera A, Hure AJ, McEvoy M, Attia J. A systematic review and meta-analysis of dietary patterns and depression in community-dwelling adults. Am J Clin Nutr. 2014;99(1):181-97.

30. Opie RS, Itsiopoulos C, Parletta N, Sanchez-Villegas A, Akbaraly TN, Ruusunen $A$, et al. Dietary recommendations for the prevention of depression. Nutr Neurosci. 2017;20(3):161-71.

31. Pourmotabbed A, Moradi S, Babaei A, Ghavami A, Mohammadi H, Jalili C, et al. Food insecurity and mental health: A systematic review and meta-analysis. Public Health Nutr. 2020;23(10):1778-90.

32. Stogios N, Smith E, Asgariroozbehani R, Hamel L, Gdanski A, Selby P, et al. Exploring patterns of disturbed eating in psychosis: A scoping review. Nutrients. 2020;12(12):3883.

33. Fountaine RJ, Taylor AE, Mancuso JP, Greenway FL, Byerley LO, Smith SR, et al. Increased food intake and energy expenditure following administration of olanzapine to healthy men. Obes (Silver Spring). 2010;18(8):1646-51.

34. Kluge M, Schuld A, Himmerich H, Dalal M, Schacht A, Wehmeier PM, et al. Clozapine and olanzapine are associated with food craving and binge eating: Results from a randomized double-blind study. J Clin Psychopharmacol. 2007;27(6):662-6. 
35. Collins SM, Surette M, Bercik P. The interplay between the intestinal microbiota and the brain. Nat Rev Microbiol. 2012;10(11):735-42.

36. Logan AC, Katzman M. Major depressive disorder: Probiotics may be an adjuvan therapy. Med Hypotheses. 2005;64(3):533-8.

37. Mörkl S, Butler MI, Holl A, Cryan JF, Dinan TG. Probiotics and the Microbiota-GutBrain Axis: Focus on Psychiatry. Curr Nutr Rep. 2020;9(3):171-82.

38. Sarkar A, Lehto SM, Harty S, Dinan TG, Cryan JF, Burnet PWJ. Psychobiotics and the Manipulation of Bacteria-Gut-Brain Signals. Trends Neurosci. 2016;39(11):763-81.

39. Chinna Meyyappan A, Forth E, Wallace CJK, Milev R. Effect of fecal microbiota transplant on symptoms of psychiatric disorders: A systematic review. BMC Psychiatry. 2020;20(1):299

40. Cenit MC, Sanz Y, Codoñer-Franch P. Influence of gut microbiota on neuropsychiatric disorders. World J Gastroenterol. 2017;23(30):5486-98.

41. Rhee SH, Pothoulakis C, Mayer EA. Principles and clinical implications of the braingut-enteric microbiota axis. Nat Rev Gastroenterol Hepatol. 2009;6(5):306-14.

42. Kelly JR, Borre Y, O’ Brien C, Patterson E, El Aidy S, Deane J, et al. Transferring the blues: Depression-associated gut microbiota induces neurobehavioural changes in the rat. J Psychiatr Res. 2016;82:109-18.

43. Amirkhanzadeh Barandouzi Z, Starkweather AR, Henderson WA, Gyamfi A, Cong XS. Altered composition of gut microbiota in depression: A systematic review. Front Psychiatry. 2020;11:541.

44. Flowers SA, Ellingrod VL. The Microbiome in Mental Health: Potential Contribution of Gut Microbiota in Disease and Pharmacotherapy Management. Pharmacotherapy. 2015;35(10):910-6.

45. Cussotto S, Walsh J, Golubeva A V., Zhdanov A V., Strain CR, Fouhy F, et al. The gut microbiome influences the bioavailability of olanzapine in rats. EBioMedicine. 2021;66:103307

46. Zmora N, Soffer E, Elinav E. Transforming medicine with the microbiome. Sci TransI Med. 2019;11(477):eaaw1815.

47. Dash S, Clarke G, Berk M, Jacka FN. The gut microbiome and diet in psychiatry: Focus on depression. Curr Opin Psychiatry. 2015;28(1):1-6.

48. Kolodziejczyk AA, Zheng D, Elinav E. Diet-microbiota interactions and personalized nutrition. Nat Rev Microbiol. 2019;17(12):742-53.

49. Flint HJ, Duncan SH, Louis P. The impact of nutrition on intestinal bacterial communities. Curr Opin Microbiol. 2017;38:59-65.

50. Hill C, Guarner F, Reid G, Gibson GR, Merenstein DJ, Pot B, et al. Expert consensus document: The international scientific association for probiotics and prebiotics consensus statement on the scope and appropriate use of the term probiotic. Nat Rev Gastroenterol Hepatol. 2014;11(8):506-14.

51. Hemarajata P, Versalovic J. Effects of probiotics on gut microbiota: Mechanisms of intestinal immunomodulation and neuromodulation. Therap Adv Gastroenterol. 2013;6(1):39-51.

52. Gibson GR, Hutkins R, Sanders ME, Prescott SL, Reimer RA, Salminen SJ, et al. Expert consensus document: The International Scientific Association for Probiotics and Prebiotics (ISAPP) consensus statement on the definition and scope of prebiotics. Nat Rev Gastroenterol Hepatol. 2017;14(8):491-502.

53. Holscher HD. Dietary fiber and prebiotics and the gastrointestinal microbiota. Gut Microbes [Internet]. 2017;8(2):172-84. Available from: http://dx.doi.org/10.1080/1949 0976.2017 .1290756 .

54. Evrensel A, Ceylan ME. Fecal Microbiota Transplantation in the Treatment-Resistant Psychiatric Disorders. Treat Resist Psychiatry. 2018;369-76.

55. Liu RT, Walsh RFL, Sheehan AE. Prebiotics and probiotics for depression and anxiety: A systematic review and meta-analysis of controlled clinical trials. Neurosci Biobehav Rev [Internet]. 2019;102. Available from: https://doi.org/10.1016/j.neubiorev.2019.03.023. 56. Dinan TG, Stanton C, Cryan JF. Psychobiotics: A novel class of psychotropic. Biol Psychiatry. 2013;74(10):720-6.

57. Phillips JGP. The treatment of melancholia by the lactic acid bacillus. Br J Psychiatr. 1910;56(234):422-30.

58. Allen AP, Hutch W, Borre YE, Kennedy PJ, Temko A, Boylan G, et al. Bifidobacterium longum 1714 as a translational psychobiotic: Modulation of stress, electrophysiology and neurocognition in healthy volunteers. Transl Psychiatry. 2016;6:e939.

59. Akkasheh G, Kashani-Poor Z, Tajabadi-Ebrahimi M, Jafari P, Akbari H, Taghizadeh M, et al. Clinical and metabolic response to probiotic administration in patients with major depressive disorder: A randomized, double-blind, placebo-controlled trial. Nutrition. 2016;32(3):315-20.

60. Kazemi A, Noorbala AA, Azam K, Eskandari MH, Djafarian K. Effect of probiotic and prebiotic vs placebo on psychological outcomes in patients with major depressive disorder: A randomized clinical trial. Clin Nutr. 2019;38(2):522-8.

61. Nikolova VL, Cleare AJ, Young AH, Stone JM. Updated Review and Meta-Analysis of Probiotics for the Treatment of Clinical Depression: Adjunctive vs. Stand-Alone Treatment. J Clin Med. 2021;10(4):647.

62. Ghaderi A, Banafshe HR, Mirhosseini N, Moradi M, Karimi MA, Mehrzad F, et al. Clinical and metabolic response to vitamin D plus probiotic in schizophrenia patients. BMC Psychiatry. 2019;19(1):77.

63. Dickerson F, Adamos M, Katsafanas E, Khushalani S, Origoni A, Savage C, et al. Adjunctive probiotic microorganisms to prevent rehospitalization in patients with acute mania: A randomized controlled trial. Bipolar Disord. 2018;20(7):614-21. 